\title{
GERAÇÃO DE RESUMO AUTOMÁTICO DE TEXTO BASEADO EM LÓGICA FUZZY
}

\author{
Manoel Villas Bôas Júnior \\ Mestre em Computação Aplicada, ITLab/POLI da Universidade Federal do Rio de \\ Janeiro(UFRJ), Rio de Janeiro, RJ, Brasil \\ mvbjunior@poli.ufrj.br
}

Ricardo Land Rodrigues

Master Bunisses em Computação Aplicada pelo ITLab/POLI da Universidade Federal do

Rio de Janeiro (UFRJ), Rio de Janeiro, RJ, Brasil

ricardoland@gmail.com

\section{RESUMO}

Este artigo propõe a geração de resumos de texto por extração a partir dos conceitos do Paradigma Edmundsoniano onde o resumo do texto é um conjunto de suas sentenças de maior peso. $O$ peso das sentenças é calculado a partir de critérios independentes como posição das sentenças dentro do texto e determinação de palavras chaves, sendo os pesos calculados são ponderados usando lógica fuzzy. O sistema de extração proposto foi desenvolvido com a linguagem Java e as bibliotecas Lucene e jFuzzyLogic sendo as regras fuzzy especificadas na linguagem FCL. A metodologia proposta é aplicada à textos de jornais, sendo o resumo gerado avaliado usando métricas Rouge. As métricas obtidas utilizando-se lógica fuzzy foram superiores ao uso do critério de peso de palavras chaves comumente usado nos sites de resumo online.

Palavras-chave: Edmundson. Fuzzy. Resumo de Texto. Rouge. Extração de Texto.

\section{AUTOMATIC FUZZY LOGIC BASED TEXT GENERATION}

\begin{abstract}
This article proposes the generation of abstracts of text by extraction from the concepts of the Edmundsonian Paradigm where the summary of the text is a set of its sentences of greater weight. The weight of the sentences is calculated from independent criteria such as position of the sentences within the text and determination of key words, the calculated weights are weighted using fuzzy logic. The proposed extraction system was developed with the Java language and the Lucene and jFuzzyLogic libraries being the fuzzy rules specified in the FCL language. The proposed methodology is applied to newspaper texts, the generated summary being evaluated using Rouge metrics. The metrics obtained using fuzzy logic were superior to the use of the key words weight criterion commonly used in online summary sites.
\end{abstract}


Keywords: Edmundson. Fuzzy. Text Abstract. Rouge. Text Extraction.

\section{INTRODUÇÃO}

A Internet trouxe a necessidade do desenvolvimento de tecnologias relacionadas à recuperação de informação (RI) de textos em função da grande quantidade de informação disponível. Uma solução para minimizar a necessidade de leitura é o uso de técnicas de resumo automático de texto por extração onde os textos retornados da pesquisa têm seu tamanho reduzido em linhas permitindo ao usuário uma rápida leitura e escolha dos textos de maior importância. O resumo por extração é a criação de um novo texto a partir de trechos do texto original.

Este trabalho propõe um sistema de resumo de texto por extração baseado no paradigma Edmundisoniano (EDMUNDSON, 1969) com foco em textos de jornal. Foi desenvolvido um sistema em Java que extrai as sentenças do texto e calcula seus pesos utilizando-se critérios independentes como palavras chaves, identificação de entidades, posicionamento das sentenças e existência de nomes próprios. Os pesos calculados são ponderados utilizando lógica Fuzzy (KYOOMARSH; KHOSRAVI; ESLAMI; DAVOUDI, 2010) onde a máquina de inferência foi escrita com regras em alto nível em FCL (CINGOLANI; ALCALA-FDEZ, 2012) por um especialista em resumo de texto de Jornal.

O sistema desenvolvido foi utilizado na extração de resumos de textos de jornal. Estes resumos foram comparados com resumos gerados utilizando-se apenas o critério de palavras chaves. As métricas Rouge obtidas foram superiores com o uso de lógica Fuzzy. O tempo de processamento não foi significativo mostrando a aplicabilidade da ferramenta na extração de resumos para resultado de sistemas de pesquisa ou mesmo para publicação de resumos automáticos para sites online a parir de textos maiores.

\section{O PARADIGMA EDMUNDISONIANO}

Edmundson em 1969 definiu um framework para extração de resumo de texto que continua a influenciar grande parte das pesquisas na área. $O$ resumo é gerado pela concatenação das sentenças de maior peso extraídas do texto original. Edmundson definiu quatro características importantes para o cálculo do peso das sentenças. Estas características estão relacionadas às palavras das sentenças e a posição das sentenças 
no texto: peso das palavras guias (Cue words), peso das palavras de títulos (Title words), peso das palavras chaves (Keywords) e posição das sentenças no texto (Sentence Locations).

As palavras guias são palavras retiradas do corpus de um mesmo assunto, ou seja, são palavras que de alguma forma se repetem ao longo dos textos. As palavras chaves são palavras retiradas de cada texto e não do corpus, uma maneira simples de escolher as palavras chaves é verificar quantas vezes uma palavra aparece no texto. As palavras chaves serão aquelas que mais aparecem no texto e escolhidas a partir de um ponto de corte. As palavras títulos são palavras retiradas dos títulos, subtítulos e demais cabeçalhos dos textos, considera-se que o título é um "resumo" do assunto escrito pelo autor.

A localização das sentenças reflete a característica de como as pessoas escrevem os textos, segundo estudo feito por Baxendale (MANI, 2001) em 1958, 85\% da informação mais importante se encontra nas sentenças no início do parágrafo e $7 \%$ nas sentenças no final do parágrafo.

Segundo Edmundson, as características descritas acima definem um critério de extração onde pesos são atribuídos às sentenças em uma expressão Linear. O resumo é composto das sentenças de maior peso, o peso de cada um destes itens é calculado manualmente de forma empírica. Estas características são ponderadas em uma fórmula linear segundo o paradigma de Edmundson, representadas na equação 1.

$$
W(s)=\alpha C(s)+\beta K(s)+\gamma L(s)+\delta T(s)
$$

Onde:

$\mathrm{W}(\mathrm{s})=$ Peso de uma sentença em um texto

$\mathrm{S}$ = Sentença

$\mathrm{C}=$ Peso das palavras guias

$\mathrm{K}=$ Peso das palavras chaves

$\mathrm{L}=$ Posição das sentenças no texto

$T=$ Palavras Títulos

$\alpha, \beta, \gamma, \delta=$ Ponderação dos termos

A expressão proposta por Edmundson é considerada Ad hoc, isto é, uma expressão empírica, intuitiva, que permite entender as características de um resumo. Entretanto, matematicamente não define como calcular os pesos das funções e nem explica porque 
uma função linear é a melhor equação para resumo.

\section{O USO DE LÓGICA FUZZY NO PARADIGMA EDMUNDISONIANO}

A solução proposta para geração de resumos consiste na utilização de lógica Fuzzy para ponderar os critérios utilizados no cálculo do peso das sentenças. A figura 1 representa a arquitetura de um sistema Fuzzy.

Figura 1 - Arquitetura de um sistema Fuzzy

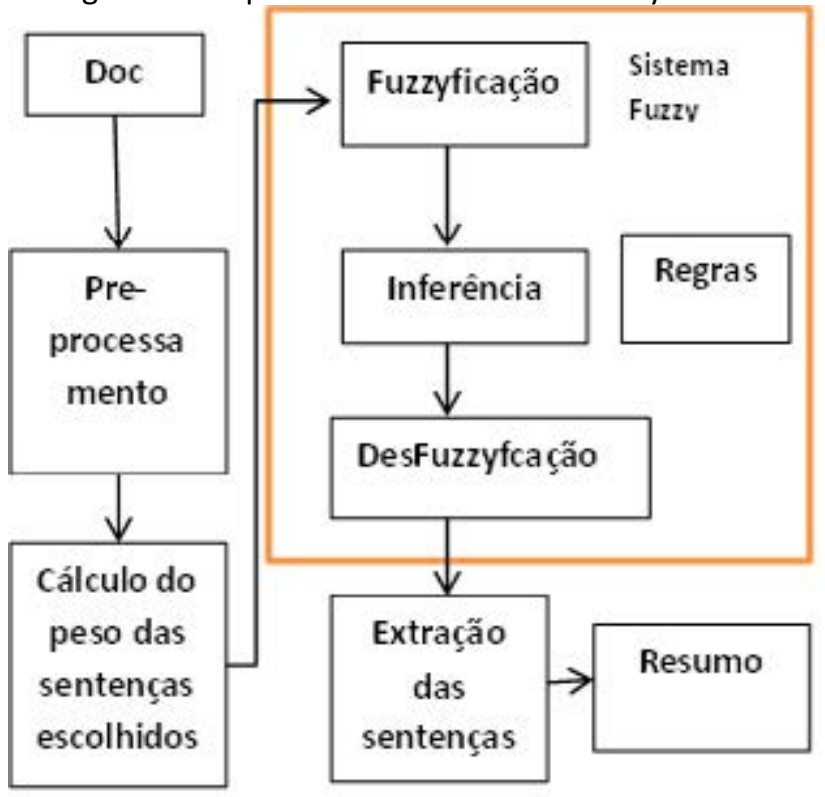

Fonte: (SUANMALI; SALIM; BINWAHLAN, 2009)

O processo de geração de resumo possui as seguintes etapas:

1. Leitura do documento a ser resumido

2. Pré-processamento do documento com extração das sentenças

3. Extração do peso das sentenças

4. Fuzzificação das características de peso em variáveis linguísticas através das

funções de pertinência

5. Aplicações das regras fuzzy (regras IF/THEN) 
6. Defuzzificação da saída retornando valor numérico para o peso das sentenças

7. Extrações das sentenças de maior peso

8. Síntese do resumo a partir das sentenças de maior peso

Os critérios de peso das sentenças escolhidos no sistema desenvolvido foram o peso das palavras chaves, posicionamento das sentenças, palavras do título, existência de números e tamanho das sentenças. $O$ sistema de extração de resumos descrito na figura 1 foi desenvolvido utilizando-se a linguagem Java e as bibliotecas Lucene para cálculo do peso das palavras chaves, jFuzzyLogic (CINGOLANI; ALCALA-FDEZ, 2012) para implementação da lógica Fuzzy e CoGrOO (KINOSHITA, SALVADOR, MENEZES, 2007) para extração das sentenças. Os demais critérios de peso das sentenças foram implementados no código sem uso de bibliotecas.

\section{FUNÇÕES DE PERTINÊNCIA}

As funções de pertinência utilizadas na extração de resumo são baseadas nas funções da tese de mestrado de Anita Ramchandara Kulkarni (KULKARNI, 2016) sendo descritas com o uso da linguagem FCL. As funções triangulares e trapezoidais foram escolhidas para representar a pertinência. São utilizadas três variáveis linguísticas para definição do peso das sentenças:

1. baixo: baixa possibilidade de um critério indicar uma sentença pertencente ao resumo.

2. médio: média possibilidade de um critério indicar uma sentença pertencente ao resumo.

3. alto: alta possibilidade de um critério indicar uma sentença pertencente ao resumo.

A figura 2 representa a função de pertinência utilizada no posicionamento das sentenças e sua especificação em FCL. 
Figura 2 - Função de pertinência Is

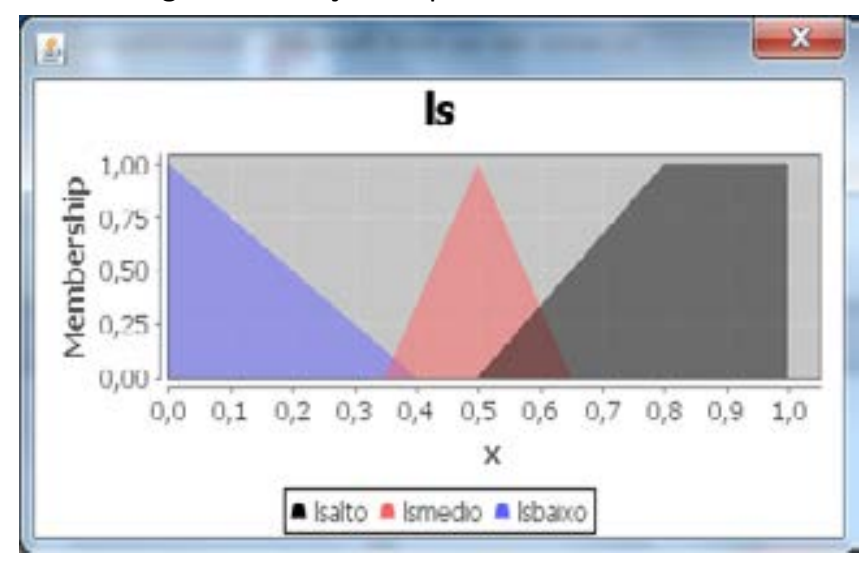

FUZZIFY Is

$$
\begin{aligned}
& \text { TERM Isalto := }(0.5,0)(0.8,1)(1,1) ; \\
& \text { TERM Ismedio:= }(0.35,0)(0.5,1.0) \quad(0.65,0.0) ; \\
& \text { TERM Isbaixo := }(0,1.0)(0.4,0.0) ; \\
& \text { END_FUZZIFY }
\end{aligned}
$$

\section{DESFUZZIFICAÇÃO}

Três variáveis linguísticas são utilizadas para defuzzificação: wsbaixo, wsmedio e wsalto

1. wsbaixo: Baixa possibilidade de uma sentença pertencer ao resumo

2. wsmedio: Média possibilidade de uma sentença pertencer ao resumo

3. wsalto: Alta possibilidade de uma sentença pertencer ao resumo

A figura 3 representa a função de pertinência escolhida para defuzzificação e sua especificação FCL. 
Figura 3 - Função de pertinência ws

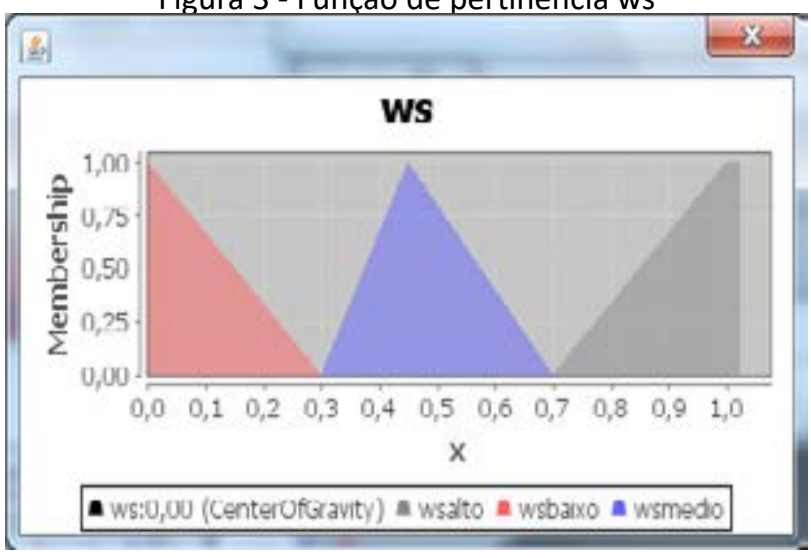

DEFUZZIFY ws

$$
\begin{aligned}
& \text { TERM wsalto }:=(0.7,0)(1,1) \\
& \text { TERM wsmedio }:=(0.3,0)(0.45,1.0)(0.7,0.0) \\
& \text { TERM wsbaixo }:=(0,1)(0.30,0) ; \\
& \text { END_DEFUZZIFY }
\end{aligned}
$$

\section{REGRAS DE INFERÊNCIA}

As regras são escritas com o uso da linguagem FCL - Fuzzy Controller Language definindo critérios para classificar as sentenças como de Alta, Média e Baixa possibilidade de pertencer ao resumo. Os seguintes conceitos foram aplicados na definição das regras de inferência:

1. Alta possibilidade de pertencer ao resumo: é considerado que se todos os critérios de entrada forem altos ou pelo menos três critérios de entrada forem altos, a sentença tem alta possibilidade de pertencer ao resumo.

2. Baixa possibilidade: é considerado que se todos os critérios de entrada forem baixos ou se apenas um dos critérios de entrada for alto, a sentença tem baixa possibilidade de pertencer ao resumo. Duas regras são acrescentadas, se a posição da sentença é baixo ou o número de palavras da sentença é baixo, a sentença tem baixa possibilidade de pertencer ao resumo. No primeiro caso é verificado que as últimas sentenças normalmente são de menor importância na leitura dos textos e no segundo caso as sentenças de menor tamanho tendem a possuir menos informação.

3. Média possibilidade: Caso a posição das sentenças e o tamanho das sentenças 
sejam altos e qualquer outro critério seja médio, a sentença tem média possibilidade de pertencer ao resumo.

As regras acima foram definidas a partir de testes feitos sobre resumo de artigos do jornal por um especialista. A figura 4 apresenta alguns exemplos de regras aplicadas escritas em FCL.

Figura 4 - Exemplo de regras

\section{RULE 2 : IF 1s IS 1salto AND ks IS ksalto THEN ws IS wsalto; RULE 3 : IF ls IS lsalto AND ns IS nsalto THEN ws IS wsalto; RULE 4: IF Is IS Isalto AND ds IS dsalto THEN ws IS wsalto; RULE 5: IF ls IS lsalto AND ts is tsalto THEN ws IS wsalto;}

\section{RESULTADOS OBTIDOS}

A metodologia proposta foi avaliada comparando-se resumos de texto feito por jornalistas e resumos feitos pela ferramenta online Tools4noobs que utiliza palavras chaves e a ferramenta desenvolvida. Os resumos gerados pelas ferramentas são comparados utilizando as métricas Rouge-1, Rouge-2 e Rouge-L (LIN, 2004). Para comparação foi utilizado o programa Rouge 2.0.

$\mathrm{Na}$ tabela I temos os valores encontrados para os critérios escolhidos para o peso das sentenças e o valor fuzzy encontrado para as duas ferramentas utilizadas. Na tabela II temos os valores Rouge calculados para cada ferramenta. O gráfico 1 faz uma comparação entre os valores encontrados. Nestas tabelas e gráfico, os valores " $\mathrm{F}$ " correspondem aos valores fuzzy e os valores " $\mathrm{T}$ " aos valores calculados pelo Tools4noobs.

Tabela I - Peso das sentenças

\begin{tabular}{|l|l|l|l|l|l|l|}
\hline $\mathbf{N}$ & ks & ts & ns & ls & ds & fz \\
\hline 1 & 0,2973 & 0,8333 & 0,2083 & 1,0000 & 0,0000 & 0,5635 \\
\hline 2 & 0,4054 & 1,0000 & 0,2500 & 1,0000 & 0,0000 & 0,6555 \\
\hline 3 & 1,0000 & 1,0000 & 0,8333 & 1,0000 & 0,0000 & 0,8997 \\
\hline 4 & 0,5946 & 0,0000 & 0,6250 & 0,5000 & 0,0000 & 0,6433 \\
\hline 5 & 0,9459 & 0,1667 & 1,0000 & 1,0000 & 0,0417 & 0,8997 \\
\hline 6 & 0,2432 & 0,1667 & 0,1667 & 1,0000 & 0,0000 & 0,1691 \\
\hline 7 & 0,7838 & 0,3333 & 0,7917 & 0,5000 & 0,0000 & 0,7872 \\
\hline 8 & 0,8919 & 0,6667 & 0,9583 & 1,0000 & 0,0000 & 0,8990 \\
\hline
\end{tabular}




\begin{tabular}{|l|l|l|l|l|l|l|}
\hline 9 & 0,5946 & 0,1667 & 0,5000 & 0,5000 & 0,0000 & 0,6205 \\
\hline 10 & 0,7297 & 0,0000 & 0,7917 & 1,0000 & 0,0000 & 0,8559 \\
\hline 11 & 0,3784 & 0,0000 & 0,4583 & 0,6667 & 0,0000 & 0,3840 \\
\hline 12 & 0,1622 & 0,0000 & 0,2500 & 0,3333 & 0,0000 & 0,1264 \\
\hline
\end{tabular}

Onde:

- $\quad \mathrm{N}$ - Número da sentença

- ks - Peso das palavras chaves do texto

- ts - Peso das palavras do título

- ns - Peso do número de palavras do texto

- Is - Peso da posição das palavras do texto

- ds - Peso dos números existente no texto

- $\mathrm{fz}$ - Peso Fuzzy resultante dos pesos anteriores

A tabela II e o gráfico 1 apresenta as métricas Rouge obtidas para o exemplo apresentado.

Tabela II - Mértricas Rouge

\begin{tabular}{|l|l|l|l|l|}
\hline Métrica & Resumo & Revocacão & Precisão & Média-F \\
\hline ROUGE-L & F & 0,38596 & 0,39286 & 0,38938 \\
\hline ROUGE-L & T & 0,37719 & 0,35246 & 0,36441 \\
\hline ROUGE-1 & F & 0,82486 & 0,86391 & 0,84393 \\
\hline ROUGE-1 & T & 0,80226 & 0,78022 & 0,79109 \\
\hline ROUGE-2 & F & 0,78916 & 0,82911 & 0,80864 \\
\hline ROUGE-2 & T & 0,75904 & 0,73684 & 0,74777 \\
\hline
\end{tabular}

Gráfico 1 - Comparação entre métricas
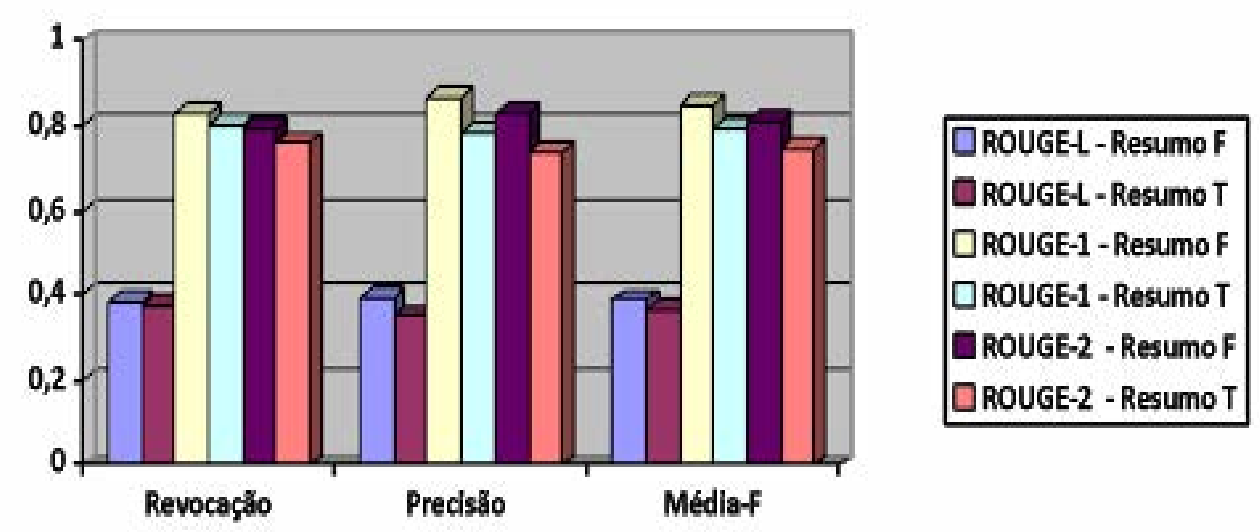

A metodologia proposta apresentou valores superiores ao uso de apenas palavras chaves e em todos os testes feitos refletiu a opinião do especialista que definiu as regras. 


\section{CONCLUSÃO}

A solução por lógica fuzzy mostrou-se rápida na extração do resumo não apresentando diferença de tempo significativa para um usuário se comparado à extração por peso de palavras chaves. Os valores das métricas Rouge foram maiores na maioria dos testes feitos. Quando os valores foram menores, verificou-se uma diferença significativa nos critérios utilizados pelo especialista que escreveu as regras de inferência e o especialista que escreveu o resumo de referência. Um critério de discordância, por exemplo, é a alto peso definido nas regras de inferência para o título do texto e alguns resumos de referência não incorporarem o título.

A utilização de diferentes critérios para o cálculo do peso das sentenças caracterizou um método incremental e simples no sentido que diferentes regras para cálculo do peso das sentenças podem ser acrescentadas e retiradas de modo a procurar uma melhor solução na extração do resumo.

\section{REFERÊNCIAS}

APACHE LUCENE. Disponível em http://lucene.apache.org/. Acesso em: 10/04/2019.

COPPIN, B. Inteligência Artificial, Editora LTC 2010, página 462.

LIN, CHIN-YEW. ROUGE: A Package for Automatic Evaluation of Summaries. Information Sciences Institute University of Southern California, 2004, Disponível em: http://www.aclweb.org/anthology/W04-1013. Acesso em: 10/04/2019.

CINGOLANI, P.; ALCALA-FDEZ, J. jFuzzyLogic: a robust and flexible Fuzzy-Logic inference system language implementation. Fuzzy Systems (FUZZ-IEEE), 2012 IEEE International Conference on. IEEE, 2012. 
EDMUNDSON, H.P, New methods in automatic abstracting, Journal of the Association for Computing Machinery, 16 (2): 264-285, 1969. Disponível em: http://courses. ischool.berkeley.edu/i256/f06/papers/edmonson69.pdf. Acesso em: 10/04/2019.

KYOOMARSH, F.; KHOSRAVI, H.; ESLAMI, E.; DAVOUDI, M. Extraction-based text summarization using Fuzzy analysis, Iranian Journal of Fuzzy System, vol 7, n0. 3, 2010 pp 15-32.

Mani, I. Automatic Summarization. John Benjamins Publishing Company. 2001.

KULKARNI, A. R., An Efficient Domain Specific Text Summarization Technique Using Knowledge Base and Combined Statistical and Linguistic Methods.Thesis. Solapur University, Chapter 6. (2016). Disponível em: https://shodhganga.inflibnet.ac.in/ handle/10603/135150. Acesso em: 10/04/2019.

SUANMALI, L.; SALIM, N.; BINWAHLAN, M.S. “Fuzzy Logic Based Method for improving text Summarization", International Journal of Computer Science and Information Security, 2009, Vol.2, No1, pp 4-10.

MEGALA S., SANTANA, KAVITHA, MARIMUTHU. Enriching text Summarization using Fuzzy Logic, International Journal of computer science and information technologies, Vol (5).1, 2014.

Summarizer Rouge. Disponível em: http://rxnlp.com/rouge-2-0/. Acesso em: 10/04/2019.

Tools you didn't even know you needed. Disponível em: https://www.tools4noobs. com/Tools4 noobs. Acesso em: 10/04/2019.

GUPTA, V.; LEHAL, G.S. A Survey of Text Summarization Extractive, Techniques Journal of Emerging Technologies in Web Intelligence, vol, 2, no, 3, August 2010. 